\title{
COMPLEX MORLET WAVELET DESIGN WITH GLOBAL PARAMETER OPTIMIZATION FOR DIAGNOSIS OF INDUSTRIAL MANUFACTURING FAULTS OF TAPERED ROLLER BEARING IN NOISYCONDITION
}

\author{
Krisztián DEÁK, Imre KOCSIS \\ University of Debrecen, Faculty of Engineering, Hungary, \\ deak.krisztian@eng.unideb.hu, kocsisi@eng.unideb.hu
}

\begin{abstract}
Detecting manufacturing defects of bearings are difficult because of their unique topography. To find adequate methods for diagnosis is important because they could be responsible for serious problems. Wavelet transform is an efficient tool for analyzing the transients in the vibration signal. In this article we are focusing on industrial grinding faults on the outer ring of tapered roller bearings. Nine different real-valued wavelets, Symlet-2, Symlet-5, Symlet-8, Daubechies (2, 6, 10, 14), Morlet and Meyer wavelets are compared to a designed complex Morlet wavelet according to the Energy-to-Shannon-Entropy ratio criteria to determine which is the most efficient for detecting the manufacturing fault. Parameters of the complex Morlet wavelet are adjustable, thus, it has more flexibility for feature extraction. Genetic algorithm is applied to optimize the center frequency and the bandwidth of the designed wavelet. A sophisticated filtering procedure through multi-resolution analysis is applied with autocorrelation enhancement and envelope detection. To determine the efficiency of the designed wavelet and compare to the other wavelets, a test-rig was constructed equipped with high-precision sensors and devices. The designed wavelet is found to be the most effective to detect the manufacturing fault. Therefore, it has the capacity for an industrial testing procedure.
\end{abstract}

Keywords: bearing vibration analysis, wavelet, optimization, genetic algorithm

\section{INTRODUCTION}

Bearing fault diagnosis is quite important in manufacturing to ensure the high quality of the product. Wavelet transform is an effective method for feature extraction and fault diagnosis in many researches.

Sawalhi and Randall determined the fault size of the bearings from the vibration signature with the entry and exit impulses of the rollers [1].

Prabhakar et al. used discrete wavelet transform with Daubechies-4 mother wavelets to analyze faults on the races of ball bearings [2].

Shi et al. applied envelope spectrum and wavelet transform together for feature extraction the defects of the bearings [3].

Nikolaou et al. applied complex Morlet wavelets for revealing vibration signals generated by rolling element bearings [4]. Complex Morlet wavelets proved to be more useful for the monitoring due to its flexibility of the center frequency and the bandwidth.

Symlet wavelets were used efficiently in the study of Kumar et al. [5]. They focused on not only to identify but measure the fault size on the outer ring from the vibration signature.

Kumar et al. executed researches with analytical wavelet transform combined with acoustic emission technique for identifying inner race faults of radial ball bearings [6].
Shi et al. used envelope analysis where the vibration signal passed through a band-pass filter to obtain a signal without significant noise then Hilbert transform was used to produce the envelope curve. The spectrum of the envelope presented useful information to reveal the fault frequencies of the bearings [7].

Honghu et al. investigated the bearing faults by using one-dimensional convolutional neural networks and long-short term memory recurrent neural networks methods by using test-rig for creating efficient training results [11].

Ivan et al. analyzed the damages of roller bearings at small turning angles. They used additional FEM analysis to model the stress conditions in bearings [12].

Kankar et al. used continuous wavelet transform for fault diagnosis of ball bearings which proved to be efficient for health monitoring in their experiments [13].

Harsha et al. applied cyclic autocorrelation combined with wavelet transform to identify ball bearing faults [14]. Cyclic autocorrelation was able to enhance the kurtosis of the signal.

Zhuang Li et al. applied wavelet transform with artificial neural network for the diagnosis of gearboxes [15].

Patel et al. used envelope analysis for the state health monitoring of deep groove ball bearings in presence of external vibration generated by Duffing 
oscillator [16]. With the envelope analysis they could reveal the bearing faults successfully.

Tabaszewski et al. concerned classification of defects of rolling bearings by k-NN (k-nearest neighbor) classifier with regard to the proper selection of the observation place. Statistical timedomain values of the vibration signal and the energy of acoustic emission pulses was found to be effective for revealing cracks in the outer rings [17].

Strączkiewicz et al. used supervised and unsupervised learning as pattern recognition methods for damage classification and clustering of rolling bearings. Clustering analysis was found to be effective for determining the bearing state conditions [18].

Wensheng et al. used optimal Morlet wavelet filter and autocorrelation enhancement to reveal bearing faults. Parameter optimization was proved to be effective for diagnosis compared to the capabilities of conventional wavelets [19].

Jena et al. investigated gear faults and identified them with analytic wavelet transform by using the vibration spectra. Envelope detection was applied to detect the signal and enhance its features for analysis [20].

Tandon et al. made a review of vibration and acoustic measurement methods for detection of defects in rolling element bearings [21].

Farzad et al. used successfully the acoustic signature extraction by wavelet transform to detect failures. Moreover, they revealed the geometrical size of the defects as well. Shannon entropy was used to determine the information content of the wavelets which helped to choose the proper wavelet for fault identification [22].

Based on the literature review, it is noticed that very limited studies on tapered roller bearing fault detection have been carried out by researches using complex Morlet wavelet, combined with the Energy-to-Shannon-Entropy ratio criteria and signal enhancement methods, especially in noisy conditions which is presented in this article.

\section{FEATURE EXTRACTION AND SIGNAI ENHANCEMENT}

\subsection{Continuous and discrete wavelet transforms}

Continuous wavelet transform (CWT) is calculated by the convolution of the signal and a wavelet function

$$
C W T_{f}(a, b)=\frac{1}{\sqrt{a}} \int_{-\infty}^{\infty} f(t) \cdot \psi^{*}\left(\frac{t-b}{a}\right) d t
$$

where $a$ is the scale parameter, $b$ is the translation parameter, $\mathrm{f}(\mathrm{t})$ is the signal in time domain, ${ }^{\psi}$ is the 'mother' wavelet, and $\psi^{*}$ is the complex conjugate of $\psi_{\text {[22]. A wavelet function as an }}$ oscillatory wave contains both the analysis and the window function.
By modifying the scale parameter, the duration and bandwidth of wavelet change provide more precise time or frequency resolution. CWT applies short windows at high frequencies and long windows at low frequencies.

CWT generates a two-dimensional map of the coefficients called scalogram

$$
S C\{f(a, b)\}=\left|C W T_{f}(a, b)\right|^{2}
$$

We define the family of functions by shifting and scaling a "mother wavelet" $\psi$

$$
\psi_{\mathrm{a}, \mathrm{b}}=\frac{1}{|\mathrm{a}|} \psi\left(\frac{\mathrm{t}-\mathrm{b}}{\mathrm{a}}\right),
$$

where $\mathrm{a}$ and $\mathrm{b}$ are reals $(a \neq 0)$, and the normalization ensures that $\left\|\psi_{a, b}(t)\right\|=\|\psi(t)\|$

Complex Morlet wavelet has the great advantage that its $\mathrm{f}_{0}$ and $\beta$ parameters can be defined separately for the certain application

$$
\psi\left(t, f_{0}, \beta\right)=\frac{1}{\sqrt{\pi \beta}} e^{-\frac{t^{2}}{\beta}} e^{2 \pi i f_{o} t}
$$

Discrete wavelet transform (DWT) applies filter banks for the analysis and synthesis of a signal. By adopting the dyadic scale and translation in the form of discretization of continuous wavelet as follows [31]

$$
D W T_{s}(j, k)=\frac{1}{\sqrt{2^{j}}} \int_{-\infty}^{\infty} s(t) \cdot \psi^{*}\left(\frac{t-2^{j} k}{2^{j}}\right) d t,
$$

where ${ }^{j}$ and $k$ are integers, $2^{j}$ and $2^{j} k$ represent the scale and translation parameters, the computational time can be reduced. The original signal $s(t)$ passes through a combination of lowpass and high-pass filters emerging as low frequency (approximations, ${ }^{a_{i}}$ ) and high frequency (details, $d_{i}$ ) signals at each decomposition level $\mathrm{i}$, called multiresolution analysis (MRA). Thus, the original signal $s(t)$ can be written as [4]

$$
\mathrm{s}(\mathrm{t})=\mathrm{a}_{\mathrm{n}}+\sum_{\mathrm{i}} \mathrm{d}_{\mathrm{i}}
$$

Using the wavelet function $\psi$ and scaling function $\varphi$ we have

$$
\begin{aligned}
& \psi_{\mathrm{j}, \mathrm{k}}[\mathrm{t}]=2^{\frac{\mathrm{j}}{2}} \sum_{\mathrm{n}} \mathrm{d}_{\mathrm{j}, \mathrm{n}} \psi\left[2^{\mathrm{j}} \mathrm{t}-\mathrm{k}\right] \\
& \varphi_{\mathrm{j}, \mathrm{k}}[\mathrm{t}]=2^{\frac{\mathrm{j}}{2}} \sum_{\mathrm{n}} \mathrm{c}_{\mathrm{j}, \mathrm{n}} \varphi\left[2^{\mathrm{j}} \mathrm{t}-\mathrm{k}\right]
\end{aligned}
$$

where $d_{j, n}$ and $c_{j, n}$ are the wavelet and scaling coefficients at scale $j$ [28]. Considering signal $X[t]=\left(v_{0}, \ldots, v_{N-1}\right)$, where $\mathrm{N}$ is the sampling number, $X_{j}[t]$ can be decomposed to scale ${ }^{j-1}$ as [30] 


$$
\begin{aligned}
& D W T \quad\left(X_{j}[t]\right) \\
& =2^{\frac{j-1}{2}} \cdot\left(\sum c A_{j-1, k} \varphi\left[2^{j-1} t-k\right]+\sum c D_{j-1, k} \psi\left[2^{j-1} t-k\right]\right),(9) \\
& 0 \leq k \leq \frac{N}{2^{j}}-1,
\end{aligned}
$$

where

$$
\begin{aligned}
& c A_{j-l, k}=\sum_{h=0}^{\frac{N}{2^{j}}-1} c_{j, h} v_{j, h+2 n}, \quad c D_{j-l, k}=\sum_{h=0}^{\frac{N}{2^{j}}-1} d_{j, h} v_{j, h+2 n} \\
& d_{h}=(-1)^{h} c_{2 p-1-h}, \quad p=N / 2^{j}
\end{aligned}
$$

Generally, $a=2$ and $b=1$ in case of the multiresolution analysis (MRA) which is an application of DWT. The multi-resolution property of the wavelet analysis allows for both good time resolution at high frequencies and good frequency resolution at low frequencies.

\subsection{Optimal parameter selection of wavelets}

For wavelet parameter optimization some important requirements should be satisfied.

Wavelet is needed to satisfy the admissibility condition

$$
c_{\psi}=\int_{-\infty}^{\infty} \frac{|\Psi(\omega)|^{2}}{|\omega|} d \omega<\infty,
$$

where $\Psi$ is the Fourier transform of $\psi, \omega$ is the angular frequency. In practice, $\Psi$ will always have sufficient decay so that the admissibility condition reduces to the requirement

$$
\int_{-\infty}^{\infty} \psi(t) d t=\Psi(0)=0 .
$$

Morlet wavelet does not satisfy this zero-mean requirement. However, the mean value can become small enough if the term $f_{0} / \beta$ is sufficiently large. When $f_{0} / \beta>3.5$ then

$$
\Psi(0)<4.2146 \times 10^{-8},
$$

therefore the admissibility condition is approximately satisfied [19].

The upper cut-off frequency of Morlet wavelet filter is $f_{0}+\beta / 2 \mathrm{~Hz}$. According to the Shannon Sampling Theorem, the upper cut-off frequency must satisfy the following condition

$$
f_{0}+\frac{\beta}{2}<\frac{f_{s}}{2.56},
$$

where $f_{s}$ is the sampling frequency.

The lower cut-off frequency of Morlet wavelet filter is $f_{0}-\beta / 2 \mathrm{~Hz}$. To reduce the interfering effects of the harmonics, the lower cut-off frequency has to be sufficiently large. Therefore, the lower cut-off frequency has to satisfy

$$
f_{0}-\frac{\beta}{2} \geq N \times f_{r}
$$

where $f_{r}$ is the rotational speed and $\mathrm{N}=35$ is chosen in this study.
To fully extract the impulsive feature, the bandwidth of the Morlet wavelet filter must be adequately wide. In this paper, we choose the bandwidth

$$
\beta>3 f_{b},
$$

where $f_{b}$ is the ball-pass frequency outer ring (BPFO) in this experiment, because the manufacturing fault is on the outer ring of the tapered roller bearing.

Finally, the problem of Morlet wavelet parameter optimization is summarized in the conditions:

$$
\begin{aligned}
& \quad \beta<f_{0} / 3.5 \\
& f_{0}+0.5 \beta<0.39 f_{s} \\
& f_{0}-0.5 \beta \geq 35 \times f_{r} \\
& \beta>3 f_{b} \\
& \text { Optimal }\left(f_{0}, \beta\right)=\min \left\{-\sum_{k=1}^{M} d_{k} \log d_{k}\right\},
\end{aligned}
$$

where

$$
d_{k}=C(k) / \sum_{i=1}^{M} C(i), k=1, \ldots, M
$$

is the normalized form of the wavelet coefficients [25].

\subsection{Maximum Energy-to-Shannon-Entropy ratio criteria}

For effective fault detection it is critical to find a proper wavelet that matches well with the shape of the signal at a specific scale and location. Low transform value is obtained if the signal and the wavelet do not correlate well. Visual observation of contour plots is not appropriate for adequate wavelet selection. Thus, a more sophisticated method for wavelet selection is used in this experiment.

Energy-to-Shannon-Entropy ratio is the combination of the energy and Shannon entropy content of the wavelet coefficients of the signal [32, 33] which is calculated by

$$
\xi(n)=E(n) / S(n)
$$

The energy content of the wavelet coefficients is determined as

$$
E(n)=\sum_{i=1}^{m}\left|C_{n, i}\right|^{2},
$$

where $m$ is the number of wavelet coefficients, $C_{n, i}$ is the ${ }^{i}$ th wavelet coefficient of $n$th scale.

The entropy of wavelet coefficients is calculated as

$$
S(n)=-\sum_{i=1}^{m} p_{i} \log _{2} p_{i}
$$

where $\left(p_{1}, \ldots, p_{m}\right)$ is the energy distribution of the wavelet coefficients at nth scale, defined as

$$
p_{i}=\left|C_{n, i}\right|^{2} / E(n)
$$




\subsection{Genetic algorithm for optimization}

Genetic algorithms (GAs) are commonly used to generate sufficiently precise solutions to optimization and search problems by relying on bio-inspired operators such as mutation, crossover and selection. In this study, GA is applied to optimize the Morlet wavelet filter parameters, the center frequency and the bandwidth.

For starting the GA operation, the initial population can be randomly determined (initialization of the population). The size of the genotype population is determined by experience. Here, the size of population is set to 50 according to the experiences.

The fitness function estimates how good an individual in the current population (fitness evaluation). In this case, the objective function is the E/S value. The fitness of each chromosome can be calculated by applying linear ranking of objective function value. The chromosome with higher fitness value is selected at greater probability than that with lower fitness value.

The selection operation selects two individuals. The stochastic universal selection (SUS) is applied in this study.

Crossover is a probabilistic process that exchanges information between two parent chromosomes for generating two child chromosomes. In this study a single-point crossover is applied, the typical crossover probability value $\mathrm{P}_{\mathrm{c}}=0.75$.

Mutation is used to avoid local convergence of the GA. Here, mutation occurs with typical mutation probability $\mathrm{P}_{\mathrm{m}}=0.03$.

The maximum number of generations was adopted as the termination criterion for the solution process. Number 60 is chosen in this paper.

Table 1. Parameters of GA for complex Morlet wavelet optimization

\begin{tabular}{|c|c|}
\hline Parameters & $\begin{array}{c}\text { Parameter } \\
\text { values }\end{array}$ \\
\hline Population scale & 50 \\
\hline $\begin{array}{c}\text { Probability of } \\
\text { mutation }\end{array}$ & 0.03 \\
\hline $\begin{array}{c}\text { Probability of } \\
\text { crossover }\end{array}$ & 0.75 \\
\hline $\begin{array}{c}\text { Terminal iteration } \\
\text { times }\end{array}$ & 60 \\
\hline \multicolumn{2}{|c|}{ Length of binary code } \\
\hline$f_{o}$ & 18 \\
\hline$\beta$ & 16 \\
\hline
\end{tabular}

\subsection{Autocorrelation envelope power spectrum for signal enhancement}

In order to enhance the signal in the frequency band, an autocorrelation enhancement algorithm is proposed. It enhances the involved periodic impulsive feature so periodic impulsive signal component related with bearing fault is strengthened while stochastic noise signal component is weakened. The analysis of autocorrelation is a tool for finding repeating patterns, such as the presence of a periodic signal obscured by noise.

The autocorrelation of the wavelet transform is expressed as

$$
\begin{aligned}
& r(l)=E\left[W T_{f_{0}, \beta}(k) W T_{f_{0}, \beta}^{*}(k+l)\right] \\
& l=0,1,2, \ldots ., k-1,
\end{aligned}
$$

where 1 is the lag index, $\mathrm{E}[\cdot]$ denotes the mathematical expectation operator.

Here, the autocorrelation envelope power spectrum is also created. The periodic impulsive feature in time domain is usually displayed as higher magnitudes of spectrum line and its harmonics in the autocorrelation envelope power spectrum

$$
\begin{aligned}
& R(f)=F[r(l)] \\
& P(f)=R(f) * R(f)
\end{aligned}
$$

where $\mathrm{F}[\cdot]$ denotes the Fourier transform and $\mathrm{P}(\mathrm{f})$ is the power spectrum. The envelope detection technique focuses on a narrow band range in the specified frequency band, which is useful for detecting the low-level impulses that are below the noise level in the normal spectrum. Autocorrelation-envelope analysis is a fault diagnosis method that can further suppress the noise and reserve the periodic components of vibration signals.

Hilbert transform is suitable for envelope detection. Hilbert transform of the signal $x(t)$ is defined by an integral transform

$$
H[x(t)]=\tilde{x}(t)=\pi^{-1} \int_{-\infty}^{\infty} \frac{x(\tau)}{t-\tau} d t
$$

It generates an artificial complex valued signal $\mathrm{H}[\mathrm{x}(\mathrm{t})]$ from the real valued $\mathrm{x}(\mathrm{t})$. The amplitude modulated envelope signal $\mathrm{E}(\mathrm{t})$ can be computed as

$$
E(t)=\sqrt{x(t)^{2}+\tilde{x}(t)^{2}} .
$$

\section{EXPERIMENTAL SETUP}

For the experiment, a bearing test-rig was planned and constructed equipped with highprecision measurement devices and sensors. (Fig. 12.)

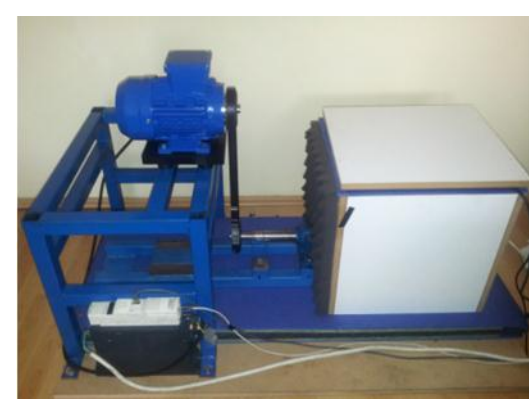

Fig. 1. Test rig for with variable speed drive tool for tapered roller bearing analysis 
The shaft is driven by an alternating current motor, the power of $0.75 \mathrm{~kW}$, frequency of $50 \mathrm{~Hz}$ and nominal speed of $2770 \mathrm{rpm}$ which is reduced to $1800 \mathrm{rpm}$ with variable speed drive device. By Schneider ATV32HU22M2 variable speed drive device the rpm can be adjusted. Constant spanning force during the measurements is measured by strain gauges in Wheatstone-bride mode on the basis of difference in voltage measurement.

NI 9234 DAQ is used in the experiment that delivers $102 \mathrm{~dB}$ of dynamic range with sampling rates up to $51.2 \mathrm{kHz}$ per channel with built-in antialiasing filters [8]. 32 bit AMD Athlon II X2 M300 $2.0 \mathrm{GHz}$ processor is used for data processing. PCB IMI 603C01 vibration transducer is applied with low noise level, high sensitivity of $100 \mathrm{mV} / \mathrm{g}$, frequency range up to $10 \mathrm{kHz}$ with top exit 2-pin connector [9]. The accelerometer is placed on the ground smooth surface $\left(\mathrm{R}_{\mathrm{a}}=1.6 \mu \mathrm{m}\right)$ of the top of the bearing house with screw that perpendicular to the axis of the rotation of the shaft. Thin layer couple fluid is applied between the bearing house and the transducer. Garant MM1-200 video microscope is used to produce magnified image about the fault and to measure its geometrical width parameter.

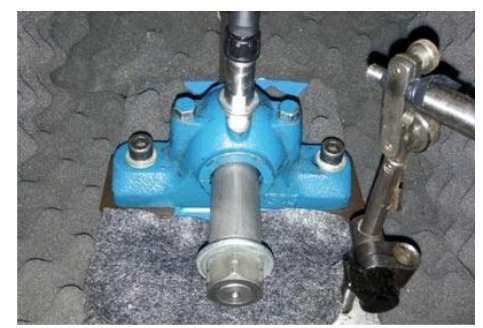

Fig. 2. Placement of the IMI 603C01 sensor on the bearing house

The shaft in the test rig is supported by two tapered roller bearings. One of the bearing is a "Type 30205" tapered roller bearing which is examined in the experiments. Four tapered roller bearings with different manufacturing defect width on the outer race (OR1-4) were measured (Table 2.).

Table 2.Geometrical parameters of grinding defects of outer rings (OR)

\begin{tabular}{|c|c|c|}
\hline Type & Width $(\mathrm{mm})$ & Depth $(\mu \mathrm{m})$ \\
\hline OR1 defect & 0.6311 & 6.5 \\
\hline OR2 defect & 1.2492 & 33.6 \\
\hline OR3 defect & 1.4751 & 42.3 \\
\hline OR4 defect & 1.6236 & 51.4 \\
\hline
\end{tabular}

Defect on the outer race is rectangular shape grinding defect (Fig. 3). The adjusted sampling frequency is $25.6 \mathrm{kHz}$ and 51200 samples are used. Due to Shannon law measurements up to $10 \mathrm{kHz}$ are valid with the sampling rate.

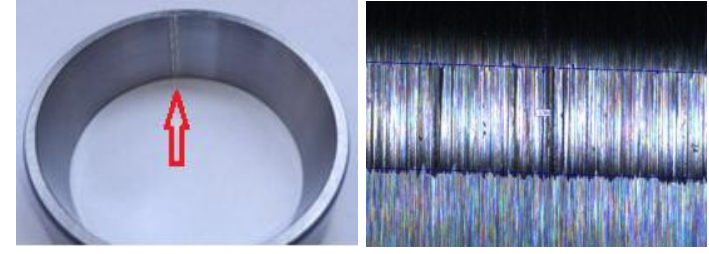

Fig. 3. Outer ring (OR2) of the tapered roller bearing with grinding defect of $1.2492 \mathrm{~mm}$ (called ORD) and its 50 times enlarged image by Garant MM1-200 video microscope

Measurements were executed in noisy environment to model the real circumstances of the bearing operation. For this purpose we used an electromechanical shaker Duffing oscillator as chaotic attractor that vibration is described by the differential equation

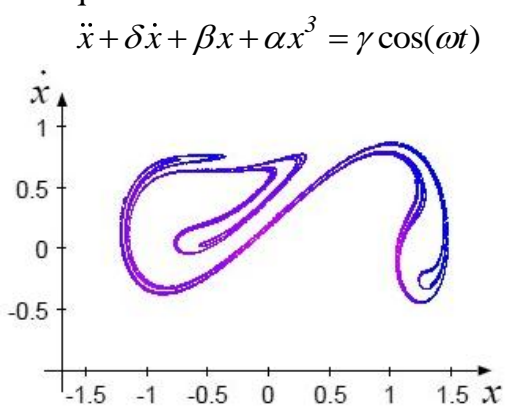

Fig. 4. Demonstration of the Duffing oscillator behaviour

\section{RESULT AND DISCUSSION}

\subsection{Parameter optimization and wavelet comparison}

With genetic algorithm the center frequency and the bandwidth of the complex Morlet wavelet (Fig. 5.) were optimized to $f_{0}=2118 \mathrm{~Hz}$ and $\beta=642 \mathrm{~Hz}$.

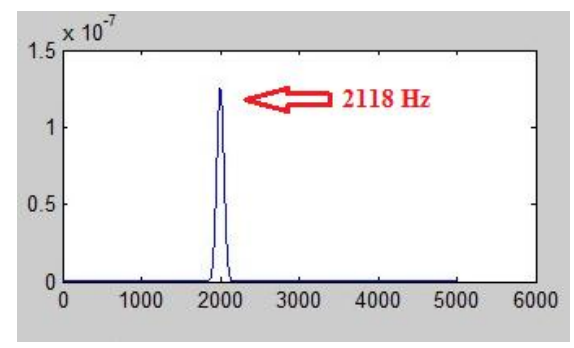

Fig. 5. The optimized complex Morlet wavelet

Ten different wavelets are considered for the present study. For the best diagnosis the wavelet with the highest $\mathrm{E} / \mathrm{S}$ ratio is obtained. The comparison of the wavelets on the basis of their E/S values are in Table 3.

Values of E/S ratio are presented graphically in Fig. 6. It is observed that the optimized complex Morlet wavelet provides the highest E/S value that indicates the Morlet wavelet to be the most efficient for both fault detection and fault size estimation. 
Table 3. Calculated values of Energy-to-ShannonEntropy ratio of the ten wavelet functions

\begin{tabular}{|l|c|c|c|c|c|}
\hline E/S & OR1 & OR2 & OR3 & OR4 & Mean \\
\hline Sym2 & 59.86 & 82.69 & 103.26 & 112.52 & 89.58 \\
\hline Sym5 & 69.58 & 98.31 & 114.01 & 112.34 & 98.56 \\
\hline Sym8 & 79.43 & 117.24 & 120.56 & 113.51 & 107.69 \\
\hline db02 & 59.86 & 82.69 & 103.26 & 112.54 & 89.59 \\
\hline db06 & 72.64 & 95.03 & 116.37 & 121.17 & 101.30 \\
\hline db10 & 77.69 & 101.8 & 121.01 & 123.05 & 105.89 \\
\hline db14 & 85.81 & 118.47 & 123.14 & 124.31 & 112.93 \\
\hline Morlet & 114.01 & 194.25 & 144.72 & 142.14 & 148.78 \\
\hline Cmor & $\mathbf{1 3 8 . 2 6}$ & $\mathbf{2 3 5 . 1 9}$ & $\mathbf{2 4 7 . 4 5}$ & $\mathbf{2 2 7 . 8 9}$ & $\mathbf{2 1 2 . 1 2}$ \\
\hline Meyer & 97.56 & 160.37 & 127.08 & 103.33 & 122.09 \\
\hline
\end{tabular}

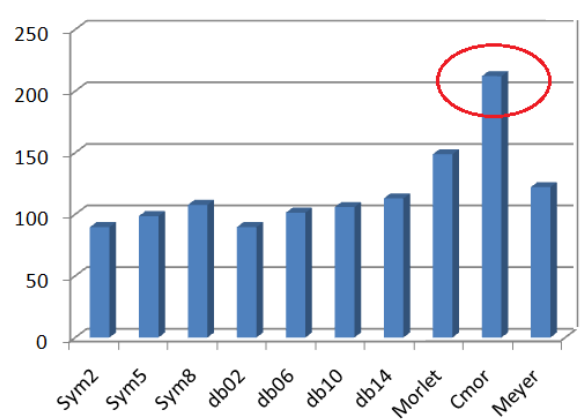

Fig. 6. Energy to Shannon Entropy ratio values of wavelet functions, $\mathrm{f}_{\mathrm{o}}=2.09 \mathrm{kHz}$

\subsection{Fault frequencies identification}

Fault frequencies are useful indicators of bearing health condition monitoring. By using the actual parameters of the bearing and operational conditions the fault frequencies are calculated. In this experiment $\mathrm{BPFO}=206.18 \mathrm{~Hz}, \mathrm{BPFI}=287.15$ $\mathrm{Hz}, \mathrm{FTF}=12.88 \mathrm{~Hz}, \mathrm{BSF}=89.96 \mathrm{~Hz}$ at $1800 \mathrm{rpm}$. Now, BPFO frequency should be used for fault detection because the manufacturing faults are on the outer ring of the bearings.

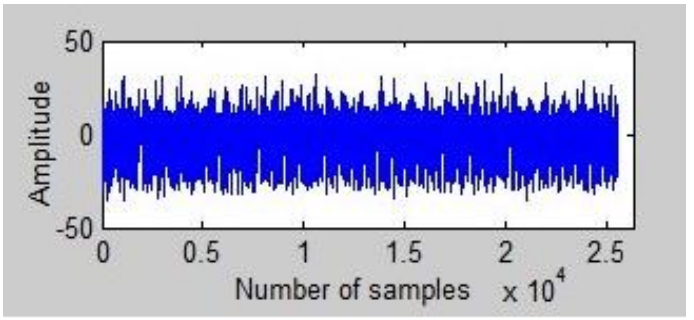

Fig. 7. Time domain raw signal of the ORD

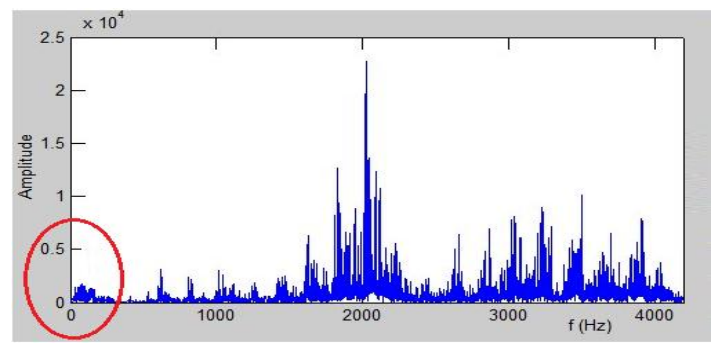

Fig. 8. FFT spectrum of the ORD

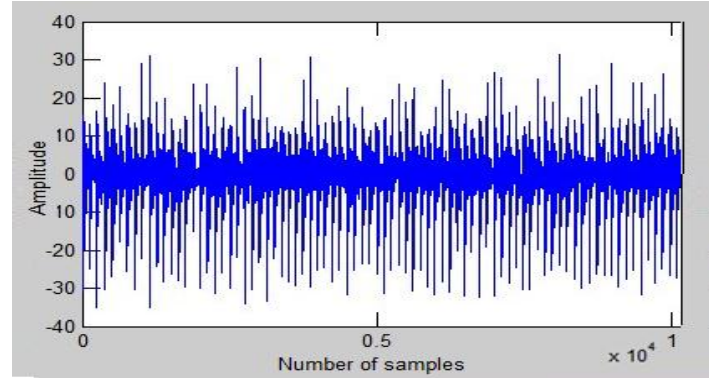

Fig. 9. Time domain spectrum of the ORD by the parameter optimized complex Morlet wavelet

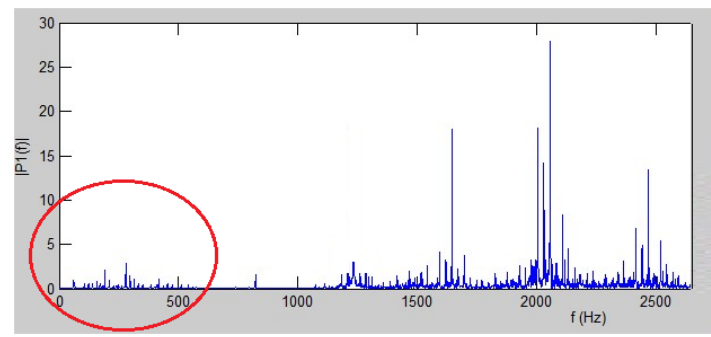

Fig. 10. FFT spectrum of the ORD filtered by the parameter optimized complex Morlet wavelet

Fig. 7. presents the raw vibration signature of outer race defect of bearing with $1.2492 \mathrm{~mm}$ fault width on its outer ring. The highest transient impulse related energy content of the burst occurs at $2.09 \mathrm{kHz}$ which is the relevant structural resonance frequency of the test-rig as a mechanical system caused by the periodic transients. The spectrum was determined on the basis of each outer ring with different fault sizes in three separate measurements for high accuracy. Each separate measurement showed similar manner and signal peak of $2.09 \mathrm{kHz}$. After filtering the raw signal by the optimized complex Morlet wavelet it is much more visible (Fig. 8.) that the outer race defect causes transients with $5 \mathrm{~ms}$ periodicity which are in connection with the calculated $206.18 \mathrm{~Hz}$ BPFO fault frequency. From FFT spectrum it is not easy to find the outer race defect frequency at $206.18 \mathrm{~Hz}$ (Fig. 8.) because of its noisy manner and low amplitude (red circle). Spectrum (Fig. 9.) offers clearer image after filtering by the optimized complex Morlet wavelet compared to the raw FFT spectrum in Fig. 8. However, it is not applicable for detailed BPFO analysis yet, because the frequency around the $206.18 \mathrm{~Hz}$ is strongly meshed (red circle) in the spectrum. Therefore, further techniques such as multi-resolution analysis, envelope spectrum and autocorrelation enhancement techniques are applied to analyze this low frequency range for precise diagnosis. 


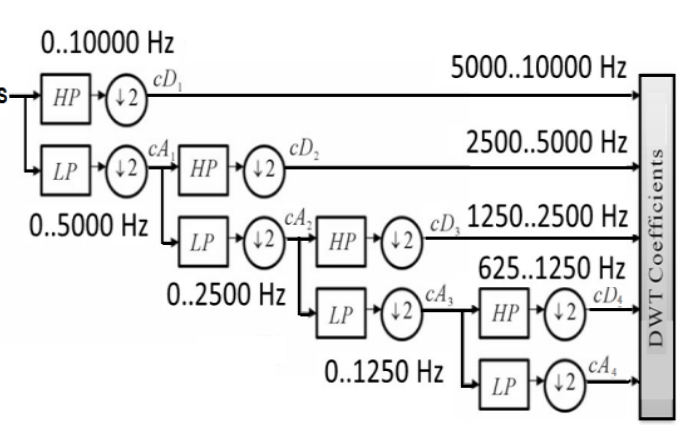

$0 . .625 \mathrm{~Hz}$

Fig. 11. Wavelet decomposition graph of the original vibration signal by MRA with the adjusted frequency ranges

Multi-resolution analysis is executed down to 4th level in order to obtain precise and detailed frequency analysis. Fig. 11-12 present the wavelet decomposition tree from signal down to the 4th level to reveal BPFO frequency for outer race grinding fault identification.

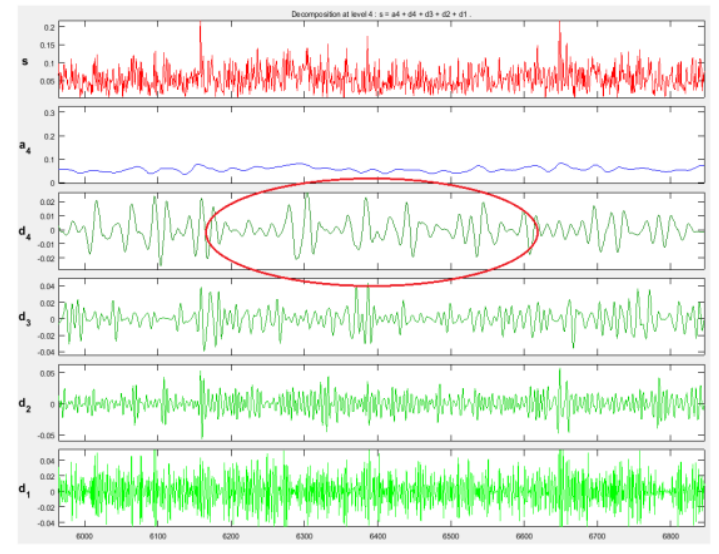

Fig.12. Multi-resolution analysis of the signal for finding transients

Fig. 13-15 show the comparison of the envelope spectrum of the bearing fault with three chosen wavelets (Symlet-5, Morlet, Cmor) from the ten wavelets for illustration. The Morlet wavelet provided better feature extraction, therefore higher BPFO peak values, than the Symlet-5 wavelet. However, the optimized complex Morlet wavelet provided the highest peak value for outer race fault identification.

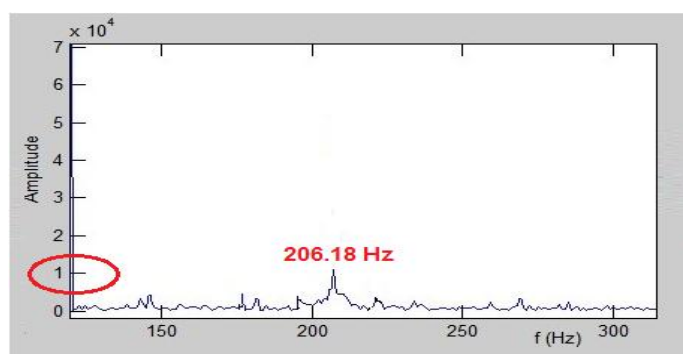

Fig.13. Envelope spectrum of the ORD filtered by Symlet- 5 wavelet

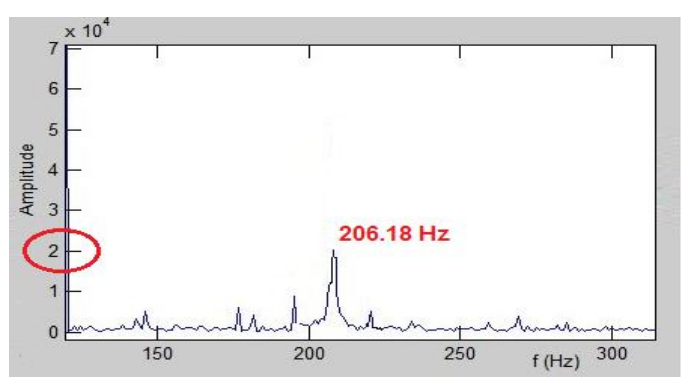

Fig.14. Envelope spectrum of the ORD filtered by Morlet wavelet

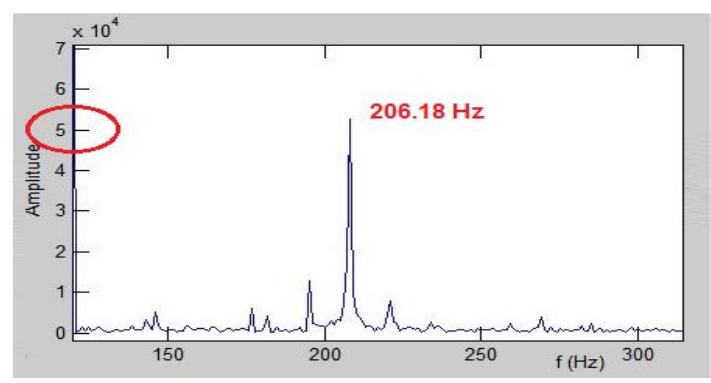

Fig. 15. Envelope spectrum of the ORD filtered by the parameter optimized Cmor wavelet

By using the autocorrelation enhancement, it is clear from Fig. 16 that the non-cyclic frequencies are suppressed in the vibration spectrum and the signal-to-noise ratio increased providing efficient fault diagnosis.

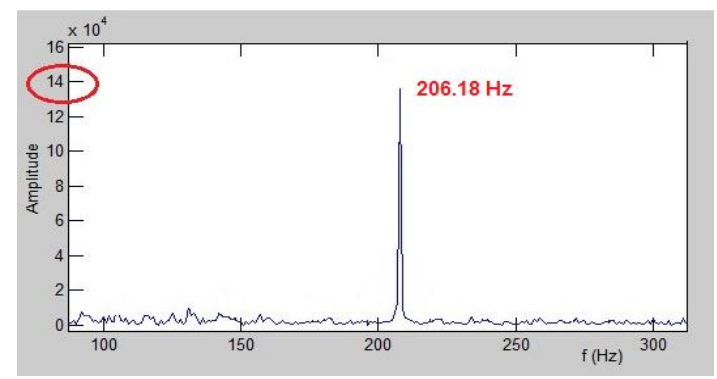

Fig. 16. Envelope spectrum of the ORD filtered by the parameter optimized Cmor wavelet with autocorrelation enhancement

Comparing the scalograms of the Symlet-5, Morlet and the optimized wavelet it is visible that the optimized complex Morlet wavelet provides the best result for diagnosis and fault size estimation (Fig. 17-19).

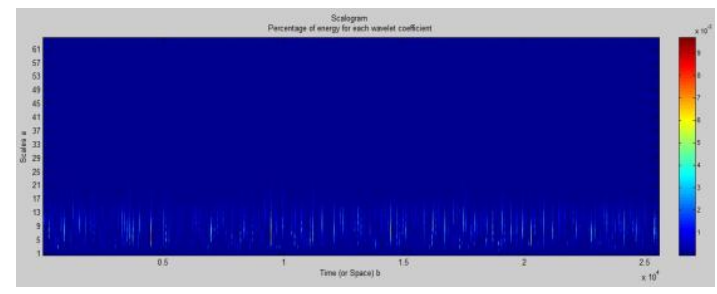

Fig. 17. Scalogram of the Symlet-5 wavelet of the ORD 


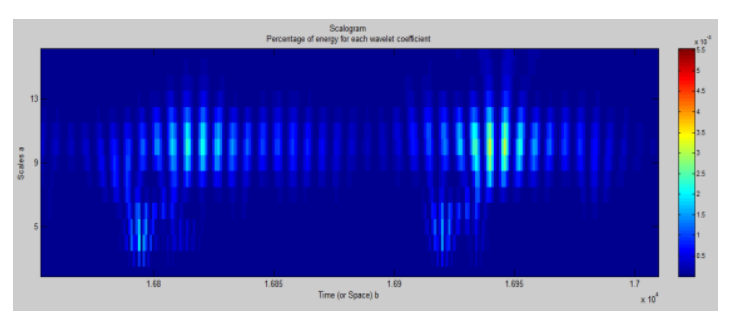

Fig. 18. Scalogram of the Morlet wavelet of the ORD

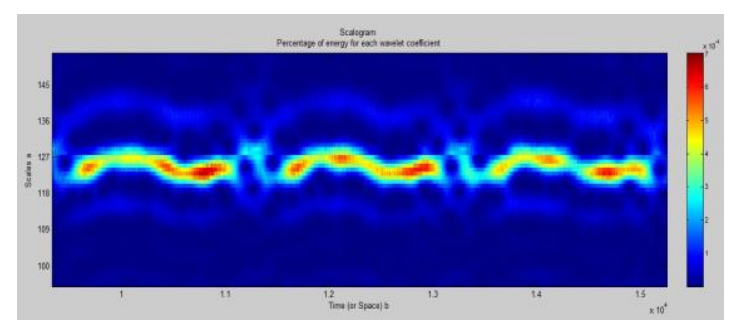

Fig. 19. Scalogram of the optimized Cmor wavelet of the ORD

\section{FAULT SIZE ESTIMATION BASED ON SCALOGRAM OF CMOR FILTER}

It is a vital task in industrial quality management to determine the fault size only from the vibration signature (without destructive method) as accurate as possible. Here, fault width was measured with high-precision laboratory devices, namely Garant MM1-200 video microscope as it is in the Table 2.

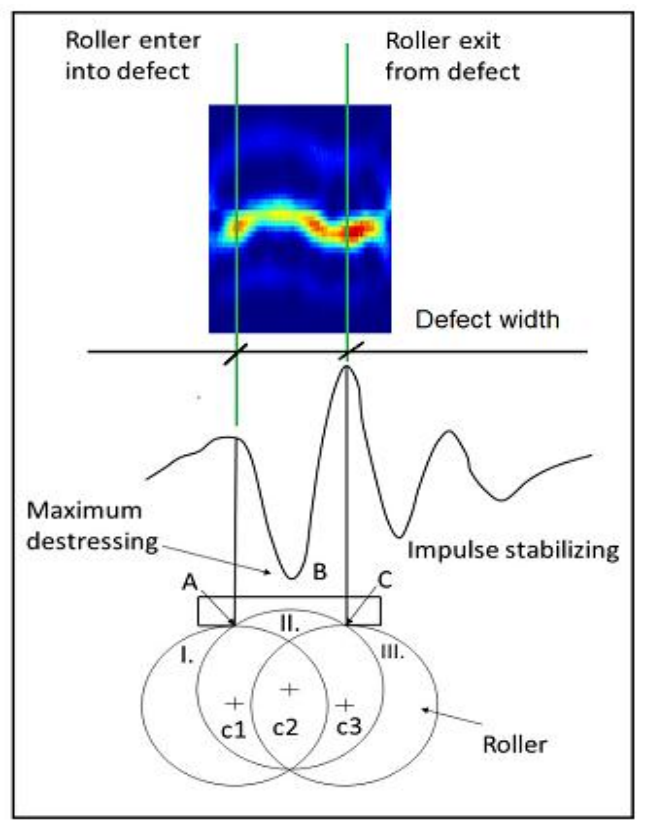

Fig. 20. Image of the optical measurement of the outer race defect width with Garant MM1-200 video microscope

Analyzing the transients, the typical points where the roller enters into and exits from the defect can be defined [5], [23].

At point $A$, the roller strikes the groove base with high impact which results in re-stressing and high impulse in signal. When the roller comes in contact with the point $B$ it again generates high amplitude in the signal and beyond the point progressive decrease in amplitude of signal is observed due to elastic damping of bearing element. At point $C$ roller comes out of the groove and creates high amplitude.

For peak detection in the scalogram, local maxima filtering algorithm and Sobel edged detection were applied which emphasize the high intensity areas (designated with red color) and found the local peaks. With this procedure entry and exit points can be exactly determined for fault width measurement. The method is equivalent with the direct finding of the maximum values in the wavelet coefficients.
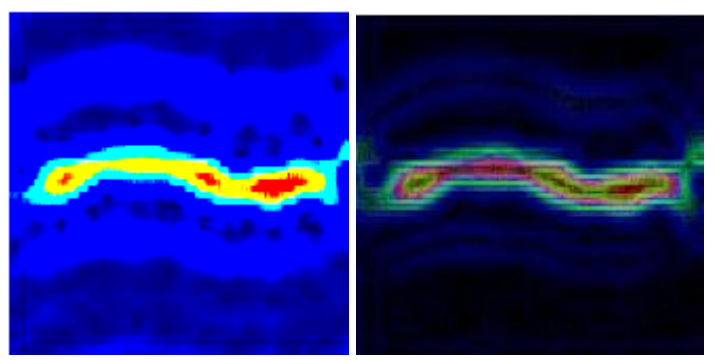

Fig. 21. Scalogram after contrast enhancement (left) and then after Sobel edge detection (right)

This technique is applicable for fault width measurement. Compared to the different wavelets (Table 3.) the results are in excellent correlation with the Energy-to-Shannon Entropy values that are calculated and displayed in Table 2. The optimized complex Morlet (Cmor) wavelet provides the most accurate result with only $1.19 \%$ deviation from the measured value by Garant MM1-200 video microscope.

Table 4. Comparison of the calculated and measured fault widths of the ORD

\begin{tabular}{|c|c|c|}
\hline Wavelet & $\begin{array}{c}\text { Calculated } \\
\text { width (mm) }\end{array}$ & $\begin{array}{c}\text { Width } \\
\text { deviation }\end{array}$ \\
\hline Sym2 & 1.277 & 2.24 \\
\hline Sym5 & 1.269 & 1.56 \\
\hline Sym8 & 1.2713 & 1.77 \\
\hline $\mathrm{db} 2$ & 1.277 & 2.24 \\
\hline $\mathrm{db} 6$ & 1.2765 & 2.19 \\
\hline $\mathrm{db} 10$ & 1.2681 & 1.52 \\
\hline $\mathrm{db} 14$ & 1.2679 & 1.50 \\
\hline Morlet & 1.266 & 1.38 \\
\hline Cmor & $\mathbf{1 . 2 6 4 0}$ & $\mathbf{1 . 1 9}$ \\
\hline Meyer & 1.2674 & 1.46 \\
\hline
\end{tabular}

\section{CONCLUSIONS}

A diagnosis method for manufacturing fault of tapered roller bearings was presented. The article focused on the detection of grinding faults on the bearing because it is typical problem in industrial manufacturing processes. With conventional time 
domain and frequency domain techniques the vibration signal was analyzed. Measurements were executed in noisy condition, where the low signalto-noise ratio made difficult the feature extraction. To draw similarities to the real circumstances an electromechanical shaker as chaotic Duffing oscillator was applied to create additional stochastic vibration. The purpose was to obtain the useful components from the vibration spectrum and enhance them for more precise fault detection and fault width measurement. Wavelet transform was used for detecting the transients in the spectrum because it had the capability to detect the sharp edges caused by the roller and fault interaction during the rotation of the bearing. Complex Morlet wavelet was defined and its center frequency and bandwidth were optimized by genetic algorithm. The efficiency of the designed complex Morlet wavelet was compared to nine different wavelets, namely Symlet-2, Symlet-5, Symlet-8, Daubechies2, Daubechies-6, Daubechies-10, Daubechies-14, Morlet and Meyer. Energy-to-Shannon-Entropy ratio criteria was used for more effective comparison. The designed wavelet provided the highest efficiency. Without filtering, the FFT spectrum was blurred by the noise making impossible to find the local BPFO fault frequency of the defected bearing. Wavelet filtering through multi-resolution analysis down to 4th level with the best-suited wavelet provided clear vibration spectrum with less noise, higher signal-to-noise ratio. However, it was needed to further emphasize the cyclic manner of the signal by using autocorrelation enhancement. The amplitude value of the BPFO frequency improved and the noncyclic frequencies were successfully suppressed. Finally, the fault width was determined from the vibration signature by using the optimized complex Morlet wavelet. The fault width measurement procedure was executed with the other nine wavelets for the purpose to compare them to the optimized wavelet. By analyzing the spectrogram with Sobel edge detection and local maxima peak detection of the wavelet coefficients, the entry point and the exit points were exactly determined where the roller entered into and exited from the defect. Results were verified by optical measurement with high resolution Garant MM1-200 video microscope. The deviation from the optically measured width was only $1.19 \%$ in case of the optimized complex Morlet wavelet. The measurements proved its capability for industrial bearing fault diagnosis in manufacturing.

\section{REFERENCES}

1. Sawalhi N, Randall RB. Vibration response of spalled rolling element bearings: observations, simulations and signal processing techniques to track the spall size. Mechanical Systems and Signal Processing. $2011 ; 25: 846$.

https://doi.org/10.1016/j.ymssp.2010.09.009
2. Prabhakar S, Mohanty AR, Sekhar AS. Application of discrete wavelet transform for detection of ball bearing race faults. Tribology International. 2002; 35:793.

3. Shi DF, Wang WJ, Qu LS. Defect detection for bearings using envelope spectra of wavelet transform. ASME Journal of Vibration and Acoustics. 2004; 126:567.

4. Nikolaou NG, Antoniadis IA. Demodulation of vibration signals generated by defects in rolling element bearings using complex shifted Morlet wavelets. Mechanical Systems and Signal Processing. 2002; 16:677.

5. Kumar R, Singh M. Outer race defect width measurement in tapered roller bearing using discrete wavelet transform of vibration signal. Measurement. 2013; 46:537.

https://doi.org/10.1016/j.measurement.2012.08.012

6. Kumar R, Jena DP, Bains M. Identification of inner race defect in radial ball bearing using acoustic emission and wavelet analysis. Proceedings of ISMA 2010 including USD 2010 Leuven (Belgium). 2010; $2883-2891$.

7. He W, Jiang Z, Feng K. Bearing fault detection based on optimal wavelet filter and sparse code shrinkage. Measurement. 2009; 42:1092-1102.

8. NI 9234 datasheet: http://www.ni.com/datasheet/pdf/en/ds-316, accessed on 2016-02-04 .

9. PCB IMI 603C01 transducer, from https://www.pcb.com/contentstore/docs/PCB Corpor ate/IMI/Products/Manuals/603C01.pdf, accessed on 2016-02-04.

10. Misiti M, Misiti Y, Oppenheim G, Poggi JM. Wavelets and their Applications. 2007; ISTE Ltd.

11. Honghu P, Xingxi H, Sai T, Fanming M. An improved bearing fault diagnosis method using onedimensional CNN and LSTM. Strojniski vestnikJournal of Mechanical Engineering. 2018; 64:443452.

12. Ivan O, Marko N, Jernej K. Analysis on damage to rolling bearings at small turning angles. Strojniski vestnik-Journal of Mechanical Engineering. 2018; 64: 209-215.

13. Kankar PK, Sharma SC, Harsha SP. Fault diagnosis of ball bearings using continuous wavelet transform. Applied Soft Computing. 2011; 11:2300-2312.

14. Harsha SP, Kankar PK, Sharma SC. Fault diagnosis of rolling element bearing using cyclic autocorrelation and wavelet transform. Neurocomputing. 2013; 110:9-17. https://doi.org/10.1016/j.neucom.2012.11.012

15. Li Z, Ma Z, Liu Y, Teng W, Jiang R. Crack fault detection for a gearbox using discrete wavelet transform and an adaptive resonance theory neural network. Strojniski vestnik-Journal of Mechanical Engineering. 2015; 61:63-73.

16. Patel VN, Tandon N, Pandey RK. Defect detection in deep groove ball bearing in presence of external vibration using envelope analysis and Duffing oscillator. Measurement. 2012; 45:960-970. https://doi.org/10.1016/j.measurement.2012.01.047

17. Tabaszewski, M. Optimization of a nearest neighbors classifier for diagnosis of condition of rolling bearings. Diagnostyka. 2014; 15: 37-42.

18. Strączkiewicz M, Czop P, Barszcz T. Supervised and unsupervised learning process in damage 
classification of rolling element bearings. Diagnostyka. 2016; 17:71-80.

19. Wensheng S, Fengtao W, Hong Z, Zhixin Z, Zhenggang G. Rolling element bearing fault diagnosis based on optimal Morlet wavelet filter and autocorrelation enhancement. Mechanical Systems and Signal Processing. 2009; 24:1458-1472.

20. Jena DP, Panigrahi S, Kumar R. Gear fault identification and localization using analytic wavelet transform of vibration signal. Measurement, 2013; 46:1115-1124.

https://doi.org/10.1016/j.measurement.2012.11.010

21. Tandon N, Choudhury A. A review of vibration and acoustic measurement methods for detection of defects in rolling element bearings. Tribology International. 1999; 32:469-480.

22. Farzad H, Wasim O, Mohamed S. Roller bearing acoustic signature extraction by wavelet packet transform, applications in fault detection and size estimation. Applied acoustics. 2016; 104:101-118. https://doi.org/10.1016/j.apacoust.2015.11.003

23. Deak K, Kocsis I, Mankovits T. Optimal Wavelet Selection for Manufacturing Defect Size Estimation of Tapered Roller Bearings with Vibration Measurement using Shannon Entropy Criteria. Strojniški vestnik - Journal of Mechanical Engineering. 2017; 63:3-14.

\section{Received 2019-01-17}

Accepted 2019-05-08

Available online 2019-05-10

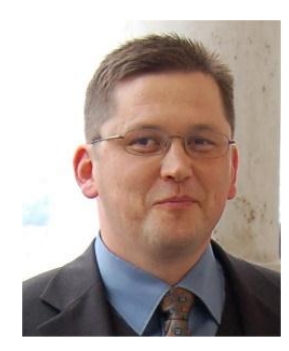

Dr. habil. Imre KOCSIS,

$\mathrm{PhD}$. is college professor at the Faculty of Engineering University of Debrecen. His scientific interests include engineering system optimization, technical diagnostics and signal processing and applied mathematical applications in engineering.

Krisztian DEAK is assistant lecturer at the Faculty of Engineering University of Debrecen. His scientific interests are related to machine fault diagnosis, modelling of dynamical systems, digital signal processing, machine learning methods and artificial intelligence applications in technical systems. 